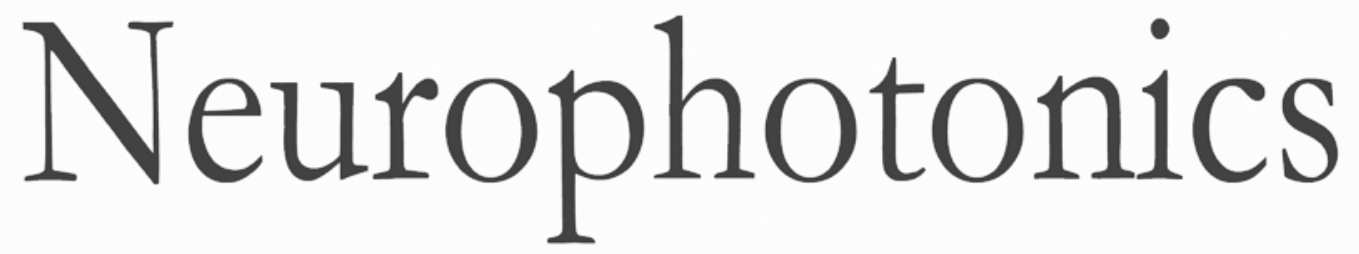

\title{
NIRS-based cerebrovascular regulation assessment: exercise and cerebrovascular reactivity
}

Stephanie Miller

Kunal Mitra 


\title{
NIRS-based cerebrovascular regulation assessment: exercise and cerebrovascular reactivity
}

\author{
Stephanie Miller, and Kunal Mitrab \\ ${ }^{a}$ Florida Institute of Technology, Department of Mechanical and Aerospace Engineering, Melbourne, Florida, United States \\ ${ }^{b}$ Florida Institute of Technology, Department of Biomedical Engineering, Melbourne, Florida, United States
}

\begin{abstract}
Alterations to cerebral blood flow (CBF) have been implicated in diverse neurological conditions. Near-infrared spectroscopy (NIRS)-measured regional cerebral tissue oxygen saturation $\left(\mathrm{rSO}_{2}\right)$ provides an estimate of oxygenation of interrogated cerebral volume useful in identifying variations in oxygen supply to cerebral tissue and in monitoring cerebrovascular function. $\mathrm{CO}_{2}$-inhalation-based hypercapnic breathing challenges were used to simulate CBF dysregulation, utilizing NIRS to monitor the CBF autoregulatory response. A breathing circuit was designed to administer $\mathrm{CO}_{2}$-compressed air mixtures and assess CBF regulatory responses to hypercapnia in 26 healthy young adults. One to three hypercapnic challenges of 5 or 10 min duration were delivered to each subject while continuously monitoring $\mathrm{rSO}_{2}$, partial pressure of end tidal $\mathrm{CO}_{2}\left(\mathrm{PETCO}_{2}\right)$, and vital signs. Change in $\mathrm{rSO}_{2}\left(\Delta \mathrm{rSO}_{2}\right)$ during $\mathrm{CO}_{2}$ inhalation positively correlated to $\Delta \mathrm{PETCO}_{2}\left(R^{2}=0.40\right)$. Grouping subjects into three exercise factor levels (h/week), (1) $0,(2)>0$ and $<10$, and (3) $>10$ showed significantly greater $\Delta \mathrm{rSO}_{2}$ responses to $\mathrm{CO}_{2}$ challenges for level 3 subjects but similar $\mathrm{PETCO}_{2}$ responses for the three groups. Exercising greater than $10 \mathrm{~h} /$ week may produce a higher resting cerebrovascular reactivity (CVR) to $\mathrm{CO}_{2}$ inhalation. Establishing baseline values of $\mathrm{rSO}_{2}$ and $\mathrm{CVR}$ to $\mathrm{CO}_{2}$ may aid in early detection of CBF changes. ๑ 2017 Society of Photo-Optical Instrumentation Engineers (SPIE) [DOI: 10.1117/1. NPh.4.4.041503]
\end{abstract}

Keywords: near-infrared spectroscopy; cerebrovascular regulation; hypercapnia; exercise.

Paper 17054SSRR received Mar. 31, 2017; accepted for publication Aug. 15, 2017; published online Sep. $12,2017$.

\section{Introduction}

Cerebrovascular regulatory control mechanisms, such as cerebrovascular reactivity (CVR) and cerebral autoregulation (CAR), respond to systemic and local triggers to control cerebral blood flow (CBF). CVR is the vasoactive response to changes in the partial pressure of the arterial carbon dioxide $\left(\mathrm{PaCO}_{2}\right.$, $\mathrm{mmHg}$ ), whereas CAR mechanisms counteract blood pressure changes in order to maintain constant CBF to the capillary beds of the brain. $\mathrm{CBF}$ dysregulation may be a biomarker of a wide variety of conditions, including mild traumatic brain injury (mTBI) and TBI. ${ }^{1,2}$ Consequences of CBF dysregulation include compromised CAR, altered glucose and oxygen metabolism and changes to cerebral perfusion pressure (CPP). Changes in $\mathrm{PaCO}_{2}$ affect $\mathrm{CBF}$ regulatory mechanisms. CVR responds to changes in $\mathrm{PaCO}_{2}$ to maintain $\mathrm{pH}$ homeostasis in the brain, which is critical to maintaining enzyme and ion channels and brain function. ${ }^{3}$ Since $\mathrm{CO}_{2}$ is a powerful vasodilator, increased $\mathrm{PaCO}_{2}$ increases $\mathrm{CBF}$ through cerebral vasodilation in healthy adults. Increased $\mathrm{PaCO}_{2}$ is also associated with a decreased rate of autoregulation (RoR), ${ }^{4}$ and breathing a $5 \% \mathrm{CO}_{2}$ mixture was found to decrease RoR by $45 \%$ as compared to normocapnia in healthy adult subjects. ${ }^{4}$

Near-infrared spectroscopy (NIRS) provides a safe (nonionizing), noninvasive method of subsurface imaging and monitoring in humans and animals that provides information about photon transport within tissues at specific wavelengths of light. Light source-detector pairs are spaced such that the path of the

*Address all correspondence to: Stephanie Miller, E-mail: mstephanie2010@ my.fit.edu
NIR light through the interrogated tissue volume will reach a specific depth range, providing information about the target area. Data collected by each source-detector pair include the time required for the light to travel from the source to the detector and the fraction of light emitted from the source that arrives at the detector. While NIRS may include measurements within the entire NIR window, applications for vascular monitoring generally use wavelengths between 700 and $900 \mathrm{~nm}^{5,6}$ Use of NIR wavelengths targeting specific properties of the absorption spectra of molecules of interest enables NIRS to provide information about the relative concentration of different species, such as oxygenated and deoxygenated hemoglobins, in the interrogated tissue volume. ${ }^{5,6}$

Regional cerebral oxygen saturation $\left(\mathrm{rSO}_{2}, \%\right)$ is a noninvasive NIRS measurement that may serve as an early warning of decreased oxygen delivery to the brain and other organs. ${ }^{7}$ This measurement is based on changes in oxygenated and deoxygenated hemoglobin contents in the interrogated tissue volume, which may reflect changes in blood flow in the region. When CBF regulatory functions are intact, periods of hypercapnia are expected to increase $\mathrm{PaCO}_{2}$ levels, resulting in cerebrovascular vasodilation, and increasing $\mathrm{rSO}_{2}$. At the same time, increases in $\mathrm{PaCO}_{2}$ can be anticipated to increase the partial pressure of end tidal $\mathrm{CO}_{2}\left(\mathrm{PETCO}_{2}, \mathrm{mmHg}\right)$, which is a noninvasive measurement that is related to but not interchangeable with $\mathrm{PaCO}_{2}{ }^{3,8,9}$ When the hypercapnic stimulus is removed, $\mathrm{PaCO}_{2}, \mathrm{PETCO}_{2}$, and $\mathrm{rSO}_{2}$ levels are expected to return to baseline values. In the case of CBF dysregulation, the $\mathrm{rSO}_{2}$ response to hypercapnia may be altered, and it is possible that $\mathrm{PaCO}_{2}$,

2329-423X/2017/\$25.00 @ 2017 SPIE 
$\mathrm{PETCO}_{2}$, and $\mathrm{rSO}_{2}$ levels may not return to baseline values upon stimulus removal.

The most accurate existing technologies for detection of $\mathrm{CBF}$ dysregulation are magnetic resonance imaging (MRI), functional MRI (fMRI), and positron emission tomography (PET). These technologies are cost prohibitive for many communities and developing countries and instrumentation and trained medical personnel are limited. As of 2013, there were 17.2 MRI machines in hospitals per 1,000,000 inhabitants in the United States. ${ }^{10}$ In France and the United Kingdom, these numbers were fewer than $10,{ }^{10}$ and in developing nations, access to state-of-the-art brain imaging technologies is scarce. Additionally, access to MRI machines is often limited by preapproval by health insurance providers, which prevents individuals who may be at risk for CBF dysregulation or have sustained mTBI from receiving diagnostic tests during the peak of the injury. Similar challenges exist with regard to fMRI and PET access. Insufficient resources exist to screen all individuals with $\mathrm{CBF}$ dysregulation risk factors. Additionally, MRI, fMRI, and PET are nonportable and footprint prohibitive for space missions and monitoring of athletes and active duty military personnel.

The different technologies used to monitor CBF or CBF correlates, including MRI, fMRI, PET, transcranial Doppler sonography (TCD), NIRS, and NIRS-based regional cerebral oximeters, all measure or calculate CBF differently and do not provide equivalent measurements. There is some question about the ability of TCD measurements to correlate well with CBF (volume/time) and CPP (pressure). ${ }^{11}$ TCD is primarily used to measure cerebral blood velocity in the form of middle cerebral artery velocity (MCAV) (distance/time). Although TCD is often used to calculate a "CVR index," this index may not be the best measure of CVR since TCD measures velocity, not flow. Additionally, TCD is ineffective in subjects with insufficient or no temporal bone window, which has been observed in $10 \%$ to $28.8 \%$ of patients ${ }^{12-14}$ and correlates to higher age, ${ }^{13,14}$ female sex, ${ }^{13,14}$ and temporal bone density, ${ }^{13}$ whereas NIRSbased $\mathrm{rSO}_{2}$ has no such limitation. No portable, inexpensive, noninvasive methods of measuring $\mathrm{CPP}, \mathrm{CBF}$, or intracranial pressure (ICP) have been accepted as benchmarks. It is critical that early shifts in CPP, CBF, CVR, and ICP be detected to implement timely countermeasures to prevent direct and persistent neurological damage.

Many factors have been correlated with CVR changes. During mild exercise, CVR increases, reaching a peak during moderate exercise, and then decreases during heavy exercise. ${ }^{8,15} \mathrm{CVR}$ demonstrates circadian rhythms and is lower in the morning than in the late afternoon and also decreases during sleep. ${ }^{16}$ An individual's CVR may change with lifetime $\mathrm{CO}_{2}$ exposure ${ }^{17}$ and may therefore be affected by age, routine exercise intensity, and long-term exposure to $\mathrm{CO}_{2}$-enriched environments. A history of brain traumas, mTBI or TBI may alter CVR. Abnormalities in CVR are implicated in sleep apnea and stroke. ${ }^{16}$ Additionally, decreased $\mathrm{CVR}^{18}$ has been reported in astronauts postflight. CAR responds to abrupt changes in blood pressure, but its ability to function during sustained changes in blood pressure and blood volume has yet to be fully characterized. It is unknown whether CAR mechanisms remain intact ${ }^{19}$ or whether CVR is altered during long duration spaceflight. Criteria defining compromised autoregulation are also lacking.

The authors' objective was to determine whether readily available, relatively inexpensive, portable, noninvasive devices that require minimal training to operate could be used to monitor CVR. First, a custom breathing circuit for the selective administration of $\mathrm{CO}_{2}$-compressed air mixtures was designed and tested in order to assess CBF regulatory responses to hypercapnic challenges in healthy young adults using a combination of noninvasive methods and real-time sensors. Next, we determined whether the particular commercial off the shelf (COTS) regional cerebral oxygenation monitor used in this study (Nonin Equanox Advance 7600 with Equanox 8004CA sensors) could be adapted for use in monitoring CVR to $\mathrm{CO}_{2}$ in the subject cohort. The use of a COTS device to monitor changes in $\mathrm{rSO}_{2}$ in response to hypercapnic challenges and correlate these responses with frequency of routine exercise in healthy young adults at rest has not been reported previously. Therefore, the relationship between CVR as measured by $\mathrm{rSO}_{2}$ and exercise frequency in healthy young adult subjects reported in this study is an unexpected result.

\section{Materials and Methods}

\subsection{Protocol and Cohort Summary}

This study had the Florida Institute of Technology (FIT) Institutional Research Board approval. ${ }^{20,21}$ FIT undergraduate and graduate student subjects were randomly selected from an open solicitation for study participants. No screening for general physical or cardiovascular fitness was used; however, all subjects selected were healthy and had no recent concussive injuries. Subjects were provided with a visual summary protocol presentation and a verbal orientation and introduction to all instrumentation used in the study and fully understood the protocol before beginning the study.

Subjects completed a basic health questionnaire, signed informed consent documents, and were weighed and their heights measured by the supervising medical professional. Subjects were asked to sit upright in an adjustable-height padded chair with armrests in a well-lit lab room, offered a blanket, and connected to the breathing circuit and instrumentation described in the following sections by the supervising medical professional.

Each study began with a 5- or 10-min baseline period during which the subjects inhaled room air through the breathing circuit. All datasets were collected during this 5- or 10-min baseline period, which was used as a reference/control. The baseline period was followed by one to three hypercapnic challenges of 5 or 10 min duration. A 5- or 10-min recovery period during which subjects breathed room air followed each hypercapnic challenge. A total of 83 challenges in 31 studies on 26 healthy, nonconcussed, FIT student subjects were delivered. Mean age (average \pm standard deviation) was $22 \pm 2.9$ year. The cohort was composed of 10 females with a height of $161.8 \pm 5.84 \mathrm{~cm}$, weight of $66.59 \pm 7.71 \mathrm{~kg}$, and BMI of $25.5 \pm 3.14$ and 16 males with a height of $176.5 \pm 7.70 \mathrm{~cm}$, weight of $74.25 \pm 18.86 \mathrm{~kg}$, and BMI of $23.7 \pm 5.22$.

\subsection{Breathing Circuit}

A breathing circuit was designed using North 5500 series halfmask respirators (Honeywell Safety Products, Smithfield, Rhode Island) connected to two 18 in. lengths of continuous positive airway pressure (CPAP) tubing (HC140C, AG Industries, St. Louis, Missouri) by in-house three-dimensional printed connectors as its base (Fig. 1). One length of CPAP 


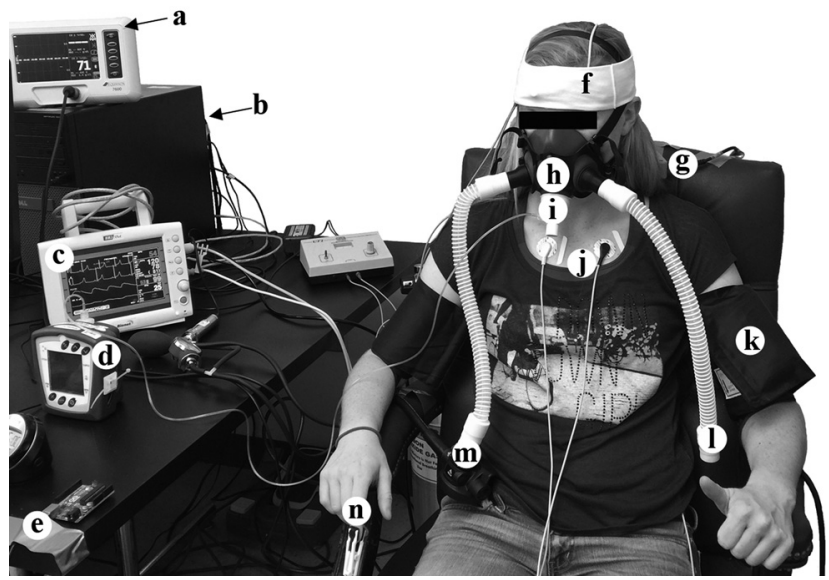

Fig. 1 Subject fully connected to breathing and monitoring circuit. (a) Nonin Equanox Advance 7600, (b) data acquisition computer, (c) Bionet BM3, (d) Smiths Medical Capnocheck II 8400, (e) Arduino Uno, (f) Nonin Equanox Advance 8004CA under cotton headband, (g) Nonin Equanox Advance 7600PA, (h) modified North 5500 halfmask respirator, (i) Smiths Medical airway adaptor/sample line, (j) ECG leads and electrodes, (k) blood pressure cuff, (I) room air end of circuit: CPAP tubing and rubber plug, $(\mathrm{m}) \mathrm{CO}_{2}$-compressed air mixed gas delivery end of circuit: SUBGEAR SG 100 and CPAP tubing, and $(\mathrm{n})$ pulse oximeter $\left(\mathrm{SpO}_{2}\right)$.

tubing was connected to a SUBGEAR SG 100 first- and secondstage regulators (SUBGEAR, Johnson Outdoors, Wendelstein, Germany). The other length of CPAP tubing was alternately plugged to close the breathing circuit and switch the inhalation source to the $\mathrm{CO}_{2}$-compressed air mixture and unplugged to open the circuit to room air. The $\mathrm{CO}_{2}$-compressed air mixture was housed in a Catalina S80 (77.4 $\mathrm{ft}^{3}$ capacity, $\left.3000 \mathrm{psi}\right)$ SCUBA cylinder, which was connected to the first-stage regulator to complete the breathing circuit. The regulator demand valve was loosened to free-float conditions to minimize the negative pressure required to draw air from the tank into the breathing circuit.

\subsection{Vital Signs}

Vital signs including heart rate and blood pressure were monitored using a Bionet BM3 (Bionet America, Inc., Tustin, California). The BM3 was connected to three electrocardiograph (ECG) leads connected to RedDot $\mathrm{Ag} / \mathrm{AgCl}$ monitoring electrodes with clear tape and solid gel (9642, 3M, St. Paul, Minnesota) in a standard Einthoven's triangle configuration, a Y-clip $\mathrm{SpO}_{2}$ sensor on the right index finger, and a blood pressure cuff on the left arm. Data for heart rate and $\mathrm{SpO}_{2}$ were acquired at a rate of $1 \mathrm{~Hz}$ using the companion B-link automated record keeping software (Bionet America, Inc., Tustin, California).

\subsection{Partial Pressure of End-Tidal $\mathrm{CO}_{2}$ and Respiration Rate}

$\mathrm{PETCO}_{2}(\mathrm{mmHg})$ and respiration rate (breaths per minute, bpm) were monitored with a Smiths Medical Capnocheck II 8400 Hand-Held Capnograph/Oximeter (Smiths Medical, St. Paul, Minnesota). The Capnocheck II provided four-breath averages for both $\mathrm{PETCO}_{2}$ and respiration rate, with an accuracy of $\pm 2 \mathrm{mmHg}$ or $4 \%$ of the reading, whichever was greater, for
$\mathrm{PETCO}_{2}$ and $\pm 1 \mathrm{bpm}$ for respiration rate. Precision was $1 \mathrm{mmHg}$ or $1 \mathrm{bpm}$.

\subsection{Regional Cerebral Oxygen Saturation}

A Nonin Equanox Advance 7600 system and 8004CA NIRS (Nonin Medical, Inc., Plymouth, Minnesota) sensor containing differentially spaced light emitters and detectors embedded in a forehead patch were used to monitor $\mathrm{rSO}_{2}$ throughout the duration of each study. The patented layout of the 76-mm-wide 8004CA sensor contained two near-infrared (NIR) light-emitting diodes with wavelengths and maximum average powers of $730 \mathrm{~nm}$ at $3.0 \mathrm{~mW}, 760 \mathrm{~nm}$ at $4.5 \mathrm{~mW}, 810 \mathrm{~nm}$ at $3.2 \mathrm{~mW}$, and $880 \mathrm{~nm}$ at $4.5 \mathrm{~mW}$ and spaced $60 \mathrm{~mm}$ apart, bookending two detection sensors spaced $20 \mathrm{~mm}$ apart. ${ }^{7,22}$ Signals from each of the two emitters were received by both a detector spaced $20 \mathrm{~mm}$ from the emitter and a detector spaced $40 \mathrm{~mm}$ from the detector. This provided two $20 \mathrm{~mm}$ paths and two $40 \mathrm{~mm}$ paths in each 8004CA sensor. ${ }^{7}$ Two sensors were used, one on each side of the forehead. The $20-\mathrm{mm}$ spacing measured extracranial tissue oxygen saturation while the $40-\mathrm{mm}$ spacing measured both extracranial and intracranial tissue oxygen saturation. ${ }^{7}$ The device uses the $20-\mathrm{mm}$ path to subtract the extracranial component of the signal from the 40-mm path in order to isolate intracranial signals. ${ }^{7}$ The purpose of using two extracranial $(20 \mathrm{~mm})$ and two overlapping extracranial + intracranial $(40 \mathrm{~mm})$ paths is to correct for differences in skin and skull optical properties between each emitter and its far $(40 \mathrm{~mm})$ detector. $^{7}$

Data were obtained for the standard 1 update per $4 \mathrm{~s}$ rate via the Equanox direct link (low resolution dataset) and were also processed by Nonin Medical, Inc. for a high-resolution dataset of 18.75 updates per second. The high-resolution dataset is reported in this paper. The manufacturer-published accuracy of the device for $\mathrm{rSO}_{2}$ values in the range of $50 \%$ to $100 \%$ was $\pm 5.1 A_{\text {rms }}$ (root mean square accuracy) absolute and $\pm 3.4 A_{\text {rms }}$ trending during hypercapnia with a sensor repeatability (precision) of \pm 2 digits of $A_{\mathrm{rms}}{ }^{21}$ The reference values used by the manufacturer for the root mean square accuracy were average oxygen saturation of arterial blood $\left(\mathrm{SavO}_{2}, \%\right)$ calculated from the average of oxygen saturation of arterial blood samples $\left(\mathrm{SaO}_{2}, \%\right)$ and oxygen saturation of jugular venous blood samples $\left(\mathrm{SjvO}_{2}, \%\right)$ as $\left.\mathrm{SavO}_{2}=\left(0.7 \times \mathrm{SjvO}_{2}\right)+\left(0.3 \times \mathrm{SaO}_{2}\right)\right)^{7,22}$

\subsection{Plethysmograph}

A custom-made plethysmograph was used to measure cuff pressure changes in the right arm at a rate of 40 samples per second.

\section{Results}

\subsection{Summary of Relationship Between $\mathrm{rSO}_{2}$ and $\triangle \mathrm{PETCO}_{2}$}

Change in $\mathrm{PETCO}_{2}\left(\triangle \mathrm{PETCO}_{2}\right)$ during $\mathrm{CO}_{2}$ inhalation was used as a correlate of the degree of hypercapnic challenge delivered. Change in $\mathrm{rSO}_{2}\left(\Delta \mathrm{rSO}_{2}\right)$ during $\mathrm{CO}_{2}$ inhalation positively correlated to $\triangle \mathrm{PETCO}_{2}\left(R^{2}=0.40\right)$. The mean response \pm standard error in $\triangle \mathrm{PETCO}_{2}$ was $6.39 \pm 0.52 \mathrm{mmHg}$, and the mean response \pm standard error in $\Delta \mathrm{rSO}_{2}$ was $2.22 \pm 0.30 \%$ (Fig. 2). 


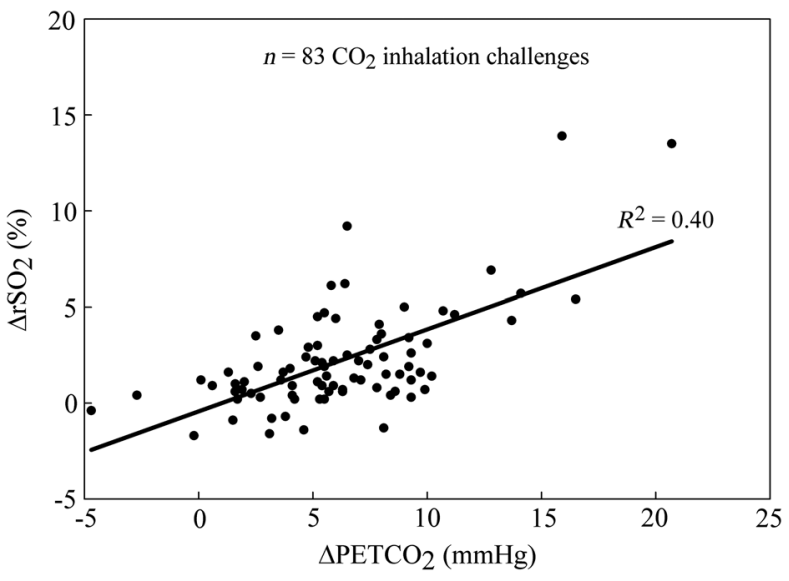

Fig. 2 Relationship between change in $\mathrm{rSO}_{2}$ versus change in $\mathrm{PETCO}_{2}$ during hypercapnia challenges as compared to prechallenge values.

\subsection{Influence of Self-Reported Exercise (h/week) on $\Delta \mathrm{rSO}_{2}$ and $\triangle \mathrm{PETCO}_{2}$}

Exercise in hours per week (h/week) was self-reported on the intake questionnaire. Subjects were grouped into three exercise factor levels as follows: (1) $0 \mathrm{~h} /$ week, (2) $>0 \mathrm{~h} /$ week and $<10 \mathrm{hr} /$ week, and $(3) \geq 10 \mathrm{~h} /$ week. Subjects grouped in exercise factor level 3 had a significantly greater mean $\Delta \mathrm{rSO}_{2}$ response to $\mathrm{CO}_{2}$ inhalation than those in exercise factor level 2 [2.82\% greater mean $\Delta \mathrm{rSO}_{2}$ response, $95 \%$ confidence interval (CI): $1.00 \%$ to $4.65 \%$ greater mean $\Delta \mathrm{rSO}_{2}$ response, $p=0.001]$ and level 1 (3.39\% greater mean $\Delta \mathrm{rSO}_{2}$ response, $95 \%$ CI: $1.67 \%$ to $5.12 \%$ greater mean $\Delta \mathrm{rSO}_{2}$ response, $p=0.00003$ ). No significant difference in subject mean $\Delta \mathrm{rSO}_{2}$ responses was observed between exercise factor levels 2 and 1 (Fig. 3).

No significant differences in mean $\triangle \mathrm{PETCO}_{2}$ were observed between the subjects in the three factor levels. This indicates that while subject $\triangle \mathrm{PETCO}_{2}$ response to $\mathrm{CO}_{2}$ inhalation did not vary with exercise factor level, $\Delta \mathrm{rSO}_{2}$ response did, and subjects exercising for greater than $10 \mathrm{~h} /$ week showed a different $\Delta \mathrm{rSO}_{2}$

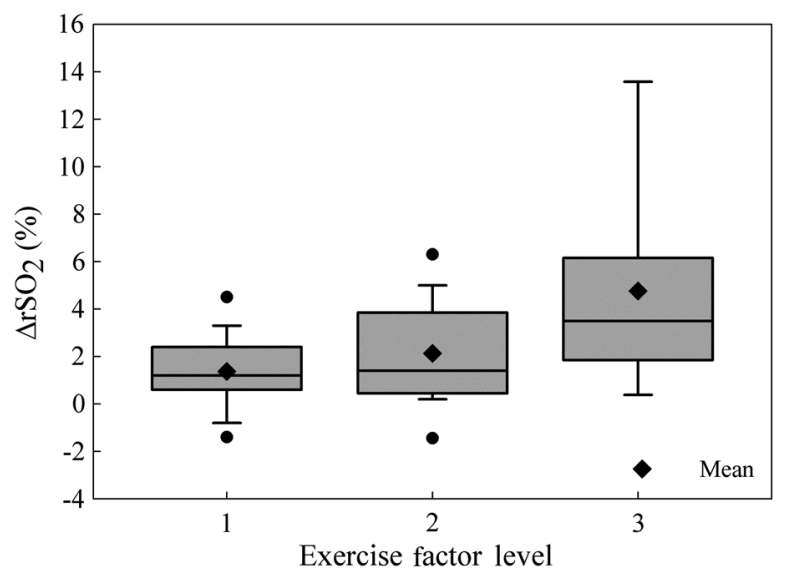

Fig. 3 Box plot of $\Delta \mathrm{rSO}_{2}$ response during $\mathrm{CO}_{2}$ challenges for exercise factor levels. Subjects grouped in exercise factor level 3 had a significantly higher $\Delta \mathrm{rSO}_{2}$ response than those in exercise factor levels $2(p=0.001)$ and $1(p=0.00003)$. Diamonds denote mean values, whiskers show 5 th/95th percentiles, and small circles show outliers.
Table 1 Subject average age, height, weight, and BMI for each exercise factor group.

\begin{tabular}{lcccc}
$\begin{array}{l}\text { Exercise } \\
\text { factor group }\end{array}$ & $\begin{array}{c}\text { Age } \\
(\text { years })\end{array}$ & $\begin{array}{c}\text { Height } \\
(\mathrm{cm})\end{array}$ & $\begin{array}{c}\text { Weight } \\
(\mathrm{kg})\end{array}$ & BMI \\
\hline 1 & $22 \pm 1.4$ & $171.1 \pm 10.28$ & $74.5 \pm 19.1$ & $25.2 \pm 4.8$ \\
2 & $23 \pm 4.5$ & $169.6 \pm 11.20$ & $68.8 \pm 13.1$ & $24.1 \pm 4.9$ \\
3 & $21 \pm 1.5$ & $173.1 \pm 3.41$ & $68.0 \pm 7.7$ & $22.8 \pm 2.6$ \\
\hline
\end{tabular}

response for the same average $\triangle \mathrm{PETCO}_{2}$ response to $\mathrm{CO}_{2}$ inhalation than subjects exercising less than $10 \mathrm{~h} /$ week. The subjects grouped in exercise level 3 demonstrated a greater CVR as measured by $\mathrm{rSO}_{2}$ than did the subjects grouped into exercise factor levels 2 and 1 . These results identify a relationship between $\mathrm{CVR}$ as measured by $\mathrm{rSO}_{2}$ in this study and exercise frequency in healthy young adults.

Self-reported exercise frequency was considered to be a more significant indicator of physical fitness than BMI because all members of exercise factor group 3 were competitive athletes. One difficulty in using this method is that individuals that may participate in activities (such as walking) at a frequency and intensity to be considered nonsedentary may self-report as sedentary. While age, height, weight, and BMI were more tightly clustered for exercise factor group 3 than for groups 1 or 2, there were no significant differences in these variables between the groups (Table 1).

Were any of these variables used to group subjects for comparison or analyzed for covariance, the large variance of these variables, particularly in exercise factor groups 1 and 2, could have erroneously categorized relatively thin, sedentary subjects in the same group as muscular, endurance trained competitive athletes. Both exercise factor groups 1 and 2 had more members with BMIs below 20 and lower minimum BMIs than exercise factor group 3 members. Many studies have investigated the relationship between physical fitness and cerebrovascular health. Age studies typically group subjects as young adult, ${ }^{17}$ middle aged, ${ }^{23}$ elderly sedentary/masters athlete, ${ }^{17}$ or simply young and older. ${ }^{24}$ All subjects in this study were young adults. Exercise studies have grouped subjects based on self-reported/ verified exercise frequency and/or type rather than BMI. ${ }^{17,23}$

\subsection{Vital Sign Results}

Continuous vital sign data were recorded for 75 of the 83 hypercapnic challenges. Data from each baseline, challenge, and recovery period were analyzed for respiration rate and heart rate against $\Delta \mathrm{rSO}_{2}$ and $\Delta \mathrm{ETCO}_{2}$, but no statistically significant relationships between these parameters could be established. Average heart rate during each postchallenge recovery period showed no significant difference from the prechallenge period in 61 of these 75 challenges. Large heart rate variability was observed. Relationships between respiration rate and other variables were complicated due to the fact that some subjects breathed spontaneously throughout the study, some paced their breathing during $\mathrm{CO}_{2}$ challenge periods and breathed spontaneously during prechallenge and recovery periods, and others paced their breathing throughout the duration of the study. 


\section{Discussion}

\subsection{Exercise and Cerebrovascular Reactivity}

The response of $\mathrm{PaCO}_{2}$ to exercise intensity is known to be parabolic. $\mathrm{PaCO}_{2}$ typically increases from low intensity through moderate intensity exercise and then decreases during heavy exercise due to hyperventilation. ${ }^{8,25}$ Rasmussen et al. proposed that $\mathrm{CBF}$ is dominantly controlled by $\mathrm{PaCO}_{2}$ and $\mathrm{CVR}$ during exercise based on the results of a 2006 study in which $\mathrm{PETCO}_{2}$, tidal volume $\left(V_{\mathrm{T}}\right)$, respiration rate, and middle cerebral artery mean velocity $\left(\mathrm{MCAV}_{\text {mean }}\right)$ were monitored using an online gas analyzer and TCD before, during, $30 \mathrm{~min}$ after, and $24 \mathrm{~h}$ after exercise on a cycle ergometer. ${ }^{25}$ The study identified a significant CVR increase above resting levels during exercise in normothermic conditions and a greater increase during exercise under hyperthermic condtions. ${ }^{25}$ The subject cohort $(n=6)$ was comprised of men ages 26 to 30 year, and $\mathrm{PaCO}_{2}$ was calculated from $\mathrm{PETCO}_{2}$ and $V_{\mathrm{T}} \cdot{ }^{25}$ A 2007 review article identified that $\mathrm{MCAV}_{\text {mean }}$ has been found to increase during exercise and subsequently decrease to resting levels upon exercise cessation, whereas MAP has been found to increase during exercise and then remain elevated above resting levels postexercise, leading to the conclusion that MAP may not be the dominant influence on increased CBF during exercise. ${ }^{8}$

Reports of the effects of age and exercise on CVR and CBF are conflicting. Thomas et al. performed a 3T MRI study and identified that masters athletes (MA) (mean age $=74$ year) had lower CVR but higher resting CBF than sedentary elderly subjects, ${ }^{17}$ which may be due to the fact that $\mathrm{PaCO}_{2}$ increases during moderate aerobic exercise ${ }^{8,15}$ and implies the possibility of a dampening of CVR due to lifetime exposure. ${ }^{17} \mathrm{Zhu}$ et al. performed a TCD study and reported no difference in CVR between MA and sedentary elderly subjects, stating "life-long aerobic exercise training appears to have minimal effects on these age-related differences in hemodynamics." ${ }^{26}$ Sedentary elderly were found to have lower baseline CBF than MA in this study. ${ }^{26}$ The discrepancy between these two studies ${ }^{17,26}$ also highlights the possibility that MRI and TCD measures of CVR may not always agree. In another TCD study that measured CVR using MCAV, previously sedentary individuals completed a 12 -week duration exercise training program. ${ }^{24}$ No information is provided to qualify "previously sedentary" with regard to lifetime activity and $\mathrm{CO}_{2}$ exposure in the elderly population; however, the young were found to have a greater response to exercise than the old. ${ }^{24}$ Many studies of the relationship of CVR to age and exercise do not consider subjects' lifetime exposure to $\mathrm{CO}_{2}{ }^{24}$

Tarumi et al. used TCD and considered three measures of CVR: hypocapnic-hypercapnic, normocapnic-hypocapnic, and normocapnic-hypercapnic. ${ }^{23}$ That study found that endurance trained middle-aged adults had significantly higher hypocapnic-hypercapnic CVR than sedentary middle-aged adults but identified no significant differences between the two groups in normocapnic-hypocapnic and normocapnic-hypercapnic CVR measures. ${ }^{23}$ In the reported study, we identified that healthy young adult athletes that exercised more than $10 \mathrm{~h} /$ week had significantly higher normocapnic-hypercapnic CVR as measured with NIRS-based $\mathrm{rSO}_{2}$ than healthy young adults who exercised $0 \mathrm{~h} /$ week and $>0$ and $<10 \mathrm{~h} /$ week, and those who exercised $>0 \mathrm{~h} /$ week and $<10 \mathrm{~h} /$ week had significantly higher normocapnic-hypercapnic CVR than those who exercised $0 \mathrm{~h} /$ week.
CVR may be dependent on numerous interacting factors, including age, exercise intensity, lifetime $\mathrm{CO}_{2}$ exposure, and other health conditions. Additionally, populations with the highest frequency of mTBI occurrences such as contactsports athletes and military personnel frequently perform moderate to heavy exercise that may alter their CVR during their lifetime. Since CBF dysfunction in the form of altered CVR may be a marker of mTBI, it may be beneficial to establish baseline CVR for all individuals likely to experience repeated or prolonged $\mathrm{CO}_{2}$ exposure or to engage in activities that may result in head trauma or that have conditions known to affect CBF or CVR and regularly monitor them for CVR changes.

\subsection{Relationship of $\mathrm{PETCO}_{2}$ to $\mathrm{PaCO}_{2}$}

Noninvasively measured $\mathrm{PETCO}_{2}$ is not interchangeable with invasively measured $\mathrm{PaCO}_{2},{ }^{3,8,9}$ and there are conflicting reports of the relationship between the two measurements. Peebles et al. identified that $\mathrm{PETCO}_{2}$ overestimates $\mathrm{PaCO}_{2}$ during normocapnia and hypercapnia ${ }^{27}$ while other studies have found $\mathrm{PETCO}_{2}$ to underestimate $\mathrm{PaCO}_{2}$ as a function of the ratio of physiologic dead space in the lungs $\left(V_{\mathrm{D}}\right)$ to $V_{\mathrm{T}}{ }^{28}$ and by as much as 13 mmHg on average. ${ }^{29}$ Regression relationships based on both $\mathrm{PETCO}_{2}$ and $V_{\mathrm{T}}^{3,25,30}$ and on $\mathrm{PETCO}_{2}$ only ${ }^{27}$ have also been published. Since the subject study only employed noninvasive methods, and $\mathrm{PaCO}_{2}, V_{\mathrm{T}}$, and $V_{\mathrm{D}}$ were not measured, no conclusions can be drawn as to the response of $\mathrm{PaCO}_{2}$ to the $\mathrm{CO}_{2}$ inhalation challenges in this study.

\section{Conclusions}

A custom breathing circuit was designed and successfully used to supply $\mathrm{CO}_{2}$-compressed air mixtures in order to assess $\mathrm{CBF}$ regulatory responses to hypercapnic challenges in healthy young adults using a COTS regional cerebral oxygenation monitor. This is the first reported use of a COTS device to monitor changes in $\mathrm{rSO}_{2}$ in response to hypercapnia and correlate these responses with frequency of routine exercise in healthy young adults at rest. The result that the subjects grouped into the highest exercise factor level ( $>10 \mathrm{~h} /$ week) had a significantly greater $\mathrm{rSO}_{2}$ but similar $\mathrm{PETCO}_{2}$ response to $\mathrm{CO}_{2}$ challenges than those grouped into exercise factor levels $2(>0 \mathrm{~h} /$ week and $<10 \mathrm{~h} /$ week $)$ or $1(0 \mathrm{~h} /$ week $)$ indicates that these young adult subjects may have had a higher CVR at rest than those who exercised less frequently or were sedentary. This relationship between CVR and exercise frequency in healthy young adult subjects as measured by $\mathrm{rSO}_{2}$ was discovered in this study.

Furthermore, the results of this study indicate that periods of $\mathrm{CO}_{2}$ inhalation may be detected by monitoring $\mathrm{rSO}_{2}$ and that cardiovascular fitness and routine exercise intensity may play a role in $\mathrm{rSO}_{2}$ response to $\mathrm{CO}_{2}$ inhalation. Establishing baseline values of $\mathrm{rSO}_{2}$ and $\mathrm{CVR}$ to $\mathrm{CO}_{2}$ may aid in early detection of $\mathrm{CBF}$ changes. These results demonstrate that it is possible to use a relatively inexpensive, noninvasive, portable device that is readily available in emergency rooms and triage units and that can be operated by a technician to monitor CVR to $\mathrm{CO}_{2}$ in real-time and in any environment. This work is significant because $\mathrm{rSO}_{2}$ monitoring is typically used in triage, emergency room, or surgical settings in order to simply identify whether brain tissue is sufficiently blood perfused. Given the broad spectrum of significance of CVR in aging, stroke, vascular, and neurological health and functioning, this study shows that it may be 
possible to monitor populations for CVR regularly without the use of expensive equipment with limited access. The minimum data sampling rate at which $\mathrm{rSO}_{2}$ can be used to monitor CVR in adults has yet to be determined.

\section{Appendix: Nonin Equanox 7600 Regional Cerebral Oximeter Details}

This study used a Nonin Equanox 7600 regional cerebral oximeter with two Equanox 8004CA sensors. This is a COTS device. Each sensor contained two light-emitting diodes emitted at four wavelengths: 730, 760, 810, and $880 \mathrm{~nm}$. Signals from each of the two emitters were received by both a detector spaced $20 \mathrm{~mm}$ from the emitter and a detector spaced $40 \mathrm{~mm}$ from the detector. This provided two $20 \mathrm{~mm}$ paths and two $40 \mathrm{~mm}$ paths in each 8004CA sensor (Fig. 4). Two sensors were used, one on each side of the forehead. The 20 -mm spacing measured extracranial tissue oxygen saturation, whereas the $40 \mathrm{~mm}$ spacing measured both extracranial and intracranial tissue oxygen saturation. ${ }^{7}$ The $20-\mathrm{mm}$ path is used to subtract the extracranial component of the signal from the $40-\mathrm{mm}$ path in order to isolate intracranial signals. The purpose of using two extracranial $(20 \mathrm{~mm})$ and two overlapping extracranial + intracranial (40 mm) paths is to correct for differences in skin and skull optical properties between each emitter and its far $(40 \mathrm{~mm})$ detector. $^{7}$

The 730,810 , and $880 \mathrm{~nm}$ wavelengths are used to calculate $\mathrm{Hb}$ and $\mathrm{HbO}_{2}$ light absorption. ${ }^{7}$ The fourth wavelength, $760 \mathrm{~nm}$, is used to compensate for skin tissue-related light absorption and reflection. ${ }^{7}$ During sensor development, atypical skin light-scattering effects were found to affect measurements when using only 730, 810, and $880 \mathrm{~nm}$, and the $760 \mathrm{~nm}$ wavelength was added to address this issue. ${ }^{7}$ The device performs internal calculations using proprietary algorithms and reports a single value for regional cerebral oxygen saturation $(\%)$.

Davie and Grocott analyzed extracranial contamination in $\mathrm{rSO}_{2}$ measurements using three popular COTS regional cerebral oximeters and found that between the three devices, the Nonin Equanox 7600 had the lowest scalp signal contamination. ${ }^{31}$ We placed an opaque, lightweight cotton headband over the two 8004CA sensors, which aided in eliminating extraneous light contamination and may have reduced scalp blood flow.

The off the shelf version of the Equanox Advance 7600 reported $\mathrm{rSO}_{2}$ one time every $4 \mathrm{~s}$. The data recorded during this study was sent to Nonin for additional high resolution processing at 18.75 samples per second.

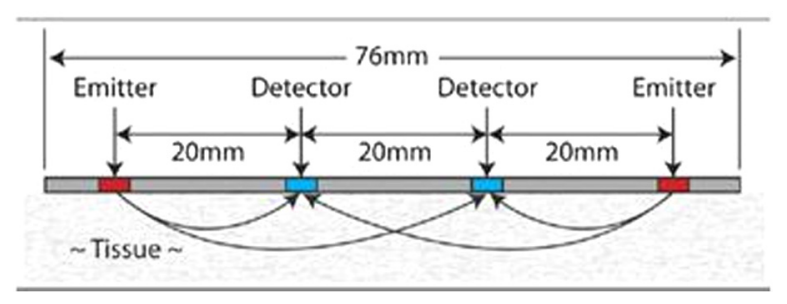

Fig. 4 Spacing of emitter and detector optodes on the Nonin Equanox 8004CA sensor (Reproduced from Fig. 1 in Ref. 7).

\section{Disclosures}

No conflicts of interest, financial or otherwise, are declared by the authors.

\section{Acknowledgments}

This work was funded by NSF CBET Grant 143551.

\section{References}

1. J. A. H. R. Classeen and R. Zhang, "Cerebral autoregulation in Alzheimer's disease," J. Cereb. Blood Flow Metab. 31, 1572-1577 (2011).

2. B. Sabayan et al., "Cerebrovascular hemodynamics in Alzheimer's disease and vascular dementia: a meta-analysis of transcranial Doppler studies," Ageing Res. Rev. 11, 271-277 (2012).

3. S. Ogoh, P. N. Ainslie, and T. Miyamoto, "Onset responses of ventilation and cerebral blood flow to hypercapnia in humans: rest and exercise," J. Appl. Physiol. 106, 880-886 (2009).

4. R. Aaslid et al., "Cerebral autoregulation dynamics in humans," Stroke 20, 45-52 (1989).

5. F. F. Jöbsis, "Noninvasive, infrared monitoring of cerebral and myocardial oxygen sufficiency and circulatory parameters," Science 198(4323), 1264-1267 (1977).

6. A. Villringer et al. "Near infrared spectroscopy (NIRS): a new tool to study hemodynamic changes during activation of brain function in adults," Neurosci. Lett. 154, 101-104 (1993).

7. D. B. MacLeod et al., "Development and validation of a cerebral oximeter capable of absolute accuracy," J. Cardiothoracic Vasc. Anesth. 26(6), 1007-1014 (2012).

8. N. H. Secher, T. Seifert, and J. Van Lieshout, "Cerebral blood flow and metabolism during exercise: implication for fatigue," J. Appl. Physiol. 104, 306-314 (2008).

9. J. A. Dempsey, P. G. Hanson, and K. S. Henderson, "Exercise-induced arterial hypoxaemia in healthy human subjects at sea level," J. Physiol. 355, 161-175 (1984).

10. Organisation for Economic Co-operation and Develepment (OECD), "Magnetic resonance imaging (MRI) units," https://data.oecd.org/ healtheqt/magnetic-resonance-imaging-mri-units.htm (06 August 2016).

11. P. Reinstrup et al., "Cerebral blood flow and transcranial doppler sonography measurements of $\mathrm{CO}_{2}$-reactivity in acute traumatic brain injured patients," Neurocrit. Care 20, 54-59 (2014).

12. C. W. A. Pennecamp et al., "The value of near-infrared spectroscopy measured cerebral oximetry during carotid endarterectomy in perioperative stroke prevention. A review," Eur. J. Vasc. Endovascular Surg. 38, 539-545 (2009)

13. A. D. Wijnhoud et al., "Inadequate acoustical temporal bone window in patients with a transient ischemic attack or minor stroke: role of skull thickness and bone density," Ultrasound Med. Biol. 34(6), 923-929 (2008).

14. Y.-P. Lin, M.-H. Fu, and T.-Y. Tan, "Factors associated with no or insufficient temporal bone window using transcranial color-coded sonography," J. Med. Ultrasound 23, 129-132 (2015).

15. C. O. Tan et al., "Cerebrovascular regulation, exercise, and mild traumatic brain injury," Neurology 83, 1665-1672 (2014).

16. K. J. Cumminds, M. Swart, and P. N. Ainslie, "Morning attenuation in cerebrovascular $\mathrm{CO}_{2}$ reactivity in healthy humans is associated with a lowered cerebral oxygenation and an augmented ventilatory response to $\mathrm{CO}_{2}$," J. Appl. Physiol. 102, 1891-1898 (2007).

17. B. P. Thomas et al., "Life-long aerobic exercise preserved baseline cerebral blood flow but reduced vascular reactivity to $\mathrm{CO}_{2}$," J. Magn. Reson. Imaging 38 1177-1183 (2013).

18. K. A. Zuj et al., "Impaired cerebrovascular autoregulation and reduced $\mathrm{CO}_{2}$ reactivity after long duration spaceflight," Am. J. Physiol. Heart Circ. Physiol. 302, H2592-H2598 (2012).

19. A. P. Blaber, K. A. Zuj, and N. Goswami, "Cerebrovascular autoregulation: lessons learned from spaceflight research,” Eur. J. Appl. Physiol. 113, 1909-1917 (2013).

20. S. Miller et al., "Development of non-invasive method for cerebrovascular regulation assessment," in Poster Presentation P-Fr-602, BMES 2015 Annual Meeting, Tampa, Florida (2015). 
21. S. Miller et al., "NIRS-based noninvasive cerebrovascular regulation assessment," Proc. SPIE 9690, 96900W (2016).

22. Nonin Medical, Inc., "Instructions for use: model 8004CA single-patient use, non-sterile, disposable regional oximetry sensor with EQUANOX ${ }^{\mathrm{TM}}$ technology," Doc. 9539-001-02, Nonin Medical, Inc., Plymouth (2014).

23. T. Tarumi et al., "Cerebral/peripheral vascular reactivity and neurocognition in middle-age athletes," Med. Sci. Sports Exercise 47(12), 25952603 (2015).

24. C. J. Murrell et al., "Cerebral blood flow and cerebrovascular reactivity at rest and during sub-maximal exercise: effect of age and 12-week exercise training," Age 35(3), 905-920 (2013).

25. P. Rasmussen et al., "Enhanced cerebral $\mathrm{CO}_{2}$ reactivity during strenuous exercise in man," Eur. J. Appl. Physiol. 96, 299-304 (2006).

26. Y.-S. Zhu et al., "Cerebral vasomotor reactivity during hypo- and hypercapnia in sedentary elderly and masters athletes," J. Cereb. Blood Flow Metab. 33, 1190-1196 (2013).

27. K. Peebles et al., "Human cerebrovascular and ventilatory $\mathrm{CO}_{2}$ reactivity to end-tidal, arterial and internal jugular vein $P_{\mathrm{CO} 2}, " J$. Physiol. 584(1), 347-357 (2007).

28. S. D. McSwain et al., "End-tidal and arterial carbon dioxide measurements correlate across all levels of physiologic dead space," Respir. Care 55(3), 288-293 (2010).
29. M. Pekdemir et al., "Disparity between mainstream and sidestream endtidal carbon dioxide values and arterial carbon dioxide levels," Respir. Care 58(7), 1152-1156 (2013).

30. N. L. Jones, D. G. Robertson, and J. W. Kane, "Difference between endtidal and arterial $\mathrm{P}_{\mathrm{CO} 2}$ in exercise," J. Appl. Physiol. 47(5), 954-960 (1979).

31. S. N. Davie and H. P. Grocott, "Impact of extracranial contamination on regional cerebral oxygen saturation," Anesthesiology 116(4), 834-840 (2012).

Stephanie Miller is a PhD candidate in the Department of Mechanical and Aerospace Engineering at Florida Institute of Technology. She holds her MS degree in mechanical engineering from Florida Institute of Technology.

Kunal Mitra is a professor in the Department of Biomedical Engineering at Florida Institute of Technology. He is a fellow of ASME and ASLMS. He is also a senior member of SPIE. He has published more than 150 peer reviewed journal papers and conference proceedings, 1 book series, 4 book chapters, and holds 2 patents. He is currently serving as an associate editor for Heat Transfer Research journal. 